\title{
INTERSTELLAR SCINTILLATION AND CLOUDS OF THE INTERSTELLAR TURBULENT PLASMA
}

\author{
V.I. SHISHOV \\ P.N. Lebedev Physical Institute, Leninskii pr. 53, 117924 Moscow, Russia
}

\begin{abstract}
Data on interstellar diffraction and refraction scintillation of pulsars are analyzed. Comparison between theory and the observational data shows that two types of spectra for electron density fluctuations are realized in the interstellar medium: pure power law and piecewise with a break. The distribution of turbulent plasma in the Galaxy has a three component structure. Component A is diffuse and it is distributed outside of the spiral arms of the Galaxy. Component BI is cloudy and associated with Galactic arms. Component BII is extremely nonuniform and associated with HII regions and supernova remnants. The origin of the interstellar plasma turbulence is considered, and possible sources of turbulent energy are discussed. The contribution of supernova bursts in the interstellar gas ionization and generation of turbulence are analyzed among other factors.
\end{abstract}

Keywords: Interstellar plasma; Scintillation; Turbulence

\section{Introduction}

In his pioneering work Scheuer (1968) supposed that pulsar intensity fluctuations, with a fine frequency structure and a temporal scale of order of minutes, are determined by scintillation on irregularities of the interstellar medium. In the next year Rickett (1969) showed that the frequency scale of the intensity fluctuations of pulsars decreases with increasing dispersion measure (DM). This result proved the interstellar origin of the pulsar flux fluctuations. More recently, Sieber (1982) revealed that characteristics of the slow pulsar flux fluctuations, with time-scales of order of days and months, depend on DM also. Large sets of data have been obtained over 30 years of investigations and we have a lot of information on the interstellar turbulent clouds which cause interstellar scintillation. However, up to now we don't have a universally accepted model of interstellar plasma turbulence.

\section{Data on Scattering and Scintillation}

The following effects of radio wave modulation by the interstellar turbulent plasma are observed:

I. Scattering effects

a) Angular scattering; the characteristic scale is $\theta_{\text {scat }}$.

b) Pulse broadening; the characteristic scale is $\tau$. 
II. Scintillation effects

c) Weak scintillation. Parameters are: $\mathrm{m}_{\text {weak }}-$ scintillation index, $\mathrm{t}_{\text {weak }}-$ characteristic temporal scale.

d) Strong or saturated scintillation

d1) Diffractive scintillation. Parameters are: $\mathrm{m}_{d i f} \cong 1-$ scintillation index, $\mathrm{t}_{\text {dif }}$ - temporal scale, $\Delta \mathrm{f}_{\text {dif }} \cong 1 / 2 \pi \tau$-frequency scale

d2) Refractive scintillation. Parameters are: $\mathrm{m}_{r e f}-$ scintillation index, $\mathrm{T}_{r e f}$ - temporal scale.

The theory of wave propagation in random media is now well developed (see, for example, Prokhorov et al., 1975; Martin and Flatte, 1988; Shishov, 1992; Smirnova, Shishov and Stinebring, 1998). For the plasma case modulation effects depend only on the power spectrum of electron density fluctuations. Theory shows that wave evolution is essentially different for three types of a turbulent spectrum (Shishov, 1993): 1) Kolmogorov type

$$
\begin{aligned}
& \Phi_{N e}(\mathbf{q})=<\left[\int d^{3} \mathbf{q} \exp (-i \mathbf{q r}) N_{e}(\mathbf{r})\right]^{2}>=C_{N e}^{2} q^{-n}, \\
& 1 / L<q<1 / l, 3<n<4,
\end{aligned}
$$

here $\mathrm{q}$ is a spatial frequency, $\mathrm{L}$ is the outer and $\mathrm{I}$ the inner scale of turbulence, $\mathrm{N}_{e}$ is electron density, $\mathrm{C}_{\mathrm{Ne}}{ }^{2}$ is a coefficient that is proportional to the square of the electron density fluctuations variance; 2) Steep power law type, that determined by equation (1) with

$$
4<\mathrm{n}<6 \text {; }
$$

and 3) one scale (Gaussian) type. Kolmogorov type spectra lead to two-scale scintillation patterns in the strong or saturated regime: small scale, or diffractive, and large scale, or refractive. The refractive scintillation index decreases with increasing distance. Steep power law spectra give a similar picture for strong scintillation, but the scintillation index of the large-scale component is constant; a large refraction angle exists for this case. The Gaussian type spectrum gives only the diffractive scintillation component in the saturated regime.

Rickett, Coles and Bourgois (1984) supposed that refractive and diffractive interstellar scintillation are caused by effects of wave propagation through a medium with a single power law (Kolmogorov) spectrum of electron density irregularities. For this spectrum and for the case of saturated scintillation one can obtain the following equations (Smirnova, Shishov and Stinebring, 1998)

$$
\begin{aligned}
& \mathrm{T}_{\text {ref }} \propto\left(\mathrm{R} \theta_{\text {scat }} / \mathrm{V}\right) \\
& \mathrm{t}_{d i f} \propto\left(1 / \mathrm{k} \theta_{\text {scat }} \mathrm{V}\right) \\
& \Delta \mathrm{f}_{\text {dif }} \propto\left(\mathrm{c} / \pi \mathrm{R} \theta_{\text {scat }}{ }^{2}\right),
\end{aligned}
$$

here $\mathrm{k}$ is wave number, $\mathrm{V}$ is the pulsar velocity, $\mathrm{c}$ is the speed of light, $\mathrm{R}$ is distance. It follows from the equations (3), that one can expect a correlation between variations of the parameters of diffractive and refractive scintillation. Analysing the 
observational data for 21 pulsars at $610 \mathrm{MHz}$, Smirnova, Shishov and Stinebring (1998) showed that the ratio of the characteristic time scales for diffractive and refractive scintillation is proportional to the decorrelation bandwidth

$$
\left(\mathrm{T}_{r e f} / \mathrm{t}_{d i f}\right) \propto \Delta \mathrm{f}_{d i f}
$$

This relation is evidence for a Kolmogorov type spectrum because this dependence follows from the equations ( 3 ). The power law index $\mathrm{n}$ can be determined by using the dependence of the refractive scintillation modulation index on the decorrelation bandwidth $\Delta \mathrm{f}_{d i f}$. Theory gives

$$
\begin{aligned}
& \mathrm{m}_{r e f}{ }^{2} \propto\left(\Delta \mathrm{f}_{d i f} / \mathrm{f}\right)^{(4-n) / 2}, \mathrm{kl} \theta_{\text {scat }}<1 \\
& \left(\Delta \mathrm{f}_{d i f} / \mathrm{f}\right)^{(4-n) / 4}, \mathrm{kl} \theta_{\text {scat }}>1,
\end{aligned}
$$

Observational data for 21 pulsars at $610 \mathrm{MHz}$ show two groups of points. Group I corresponds to a medium with a piecewise power law spectrum with the value $\mathrm{n}$ $\cong 3.67$ and inner scale $1 \cong 3 \cdot 10^{10} \mathrm{~cm}$. Group II corresponds to the pure power law spectrum with the value $\mathrm{n} \cong 3.67$. This means that the spectrum shape is different for different regions of the interstellar medium.

\section{Refractive Modulation of Diffractive Scintillation}

Theory is well developed for the case of the averaging on the total statistics. The diffractive scintillation (correlation functions) and the scattering (coherence function and pulse shape) are usually measured in the regime of partial averaging over the diffractive scintillation statistics. Measurements give the fluctuating values of visibility functions, scattered pulse and correlation functions of diffractive scintillation. First theory results predict that relative variations of the scattering and scintillation parameters should be of the order of the refractive scintillation index for the case of a Kolmogorov type spectrum (Blandford and Narayan, 1985; Shishov, 1995).

$$
\left(\delta \theta_{\text {scat }} / \theta_{\text {scaf }}\right) \approx\left(\delta \mathrm{t}_{d i f} / \mathrm{t}_{d i f}\right) \approx(\delta \tau / \tau) \approx\left(\delta \Delta \mathrm{f}_{d i f} / \Delta \mathrm{f}_{d i f}\right) \approx \mathrm{m}_{r e f}
$$

In general, observational data correspond to these predictions (Stinebring, Faison and McKinnon, 1996). For the case of a Kolmogorov turbulent spectrum, theory predicts small angle refraction

$$
\left(\delta \theta_{\text {ref }} / \theta_{\text {scul }}\right) \approx \mathrm{m}_{\text {ref }}
$$

and the shift of the maximum of the two-frequency cross-correlation function of intensity fluctuations or frequency drift. Observations show the effect of the frequency drift, that corresponds to these predictions (Gupta, Rickett and Lyne, 1994). But sometimes this effect is very strong and corresponds to very large values of the 
refraction angle, $\delta \theta_{r e f} \approx(5-10) \theta_{\text {scat }}$. It is difficult to explain such values of the refraction angle in terms of a Kolmogorov turbulent spectrum.

\section{Distribution in the Galaxy}

Cordes, Weisberg and Boriakoff (1985) established that turbulent interstellar plasma is concentrated in compact clouds and the distribution of clouds in the Galaxy has a two component structure. Component $\mathrm{A}$ is diffuse, localized in interarm regions and has a distribution that is nearly statistically uniform. Component $\mathrm{B}$ has essentially nonuniform distribution in the Galaxy and is associated with the galactic arms. It was shown by Smirnova, Shishov and Stinebring (1998) that component B can, in turn, be separated into two subcomponents. Subcomponent BI is characterized by a Kolmogorov turbulent spectrum and is distributed more uniformly in Galactic space. The spectrum of subcomponent BII can be described by a power law with inner scale $1 \approx 3 \cdot 10^{10} \mathrm{~cm}$, and this subcomponent is concentrated in compact regions adjacent to pulsars.

Pynzar' and Shishov $(1997,1999)$ investigated in more detail statistical relations between scattering and scintillation parameters on the one hand, and dispersion measure DM and emission measure EM, on the other hand. Most observational data relate to the characteristic time of pulse broadening, $\tau$, and the decorrelation bandwidth, $\Delta \mathrm{f}_{\text {dif }}$, that can be reduced to $\tau \approx 1 / 2 \pi \Delta \mathrm{f}_{\text {dif }}$. These data were reduced to frequency $\mathrm{f}=300 \mathrm{MHz}$. Observations cannot be described by a single power law .

$\tau \propto \mathrm{DM}^{\alpha}$.

For pulsars with $\mathrm{DM}<50 \mathrm{pc} / \mathrm{cm}^{3}$, the observational data correspond to the relation (8) with $\alpha \approx 2$, that is consistent with the distribution of the turbulent plasma in a statistically uniform model and corresponds to component $A$ of the distribution of the turbulent clouds in the Galaxy. For large values of dispersion measure, DM $\geq$ $50 \mathrm{pc} / \mathrm{cm}^{3}$, the dependence $\tau$ on DM corresponds to (8) with $\alpha \approx 4.5$. This dependence can be explained if electron density variation is the main factor for variations of DM and $\tau$. The region of large values of DM corresponds to component $\mathrm{B}$ of the distribution of turbulent clouds.

Some information about the nature of turbulent plasma clouds and their distribution in the Galaxy is contained in the statistical dependence of $\tau$ on emission measure, EM. Observation data can be described by the relationship (Pynzar' and Shishov, 1999)

$$
\begin{aligned}
& \tau \propto \mathrm{EM}^{2}, \mathrm{EM}<\mathrm{EM}_{c r i t} \cong 200 \mathrm{pc} / \mathrm{cm}^{3} \\
& \tau \propto \mathrm{EM}, \mathrm{EM}>\mathrm{EM}_{c r i t}
\end{aligned}
$$

Note that $\mathrm{EM} \propto \mathrm{N}_{e}{ }^{2}$ and according to Cordes and Rickett (1998), and Smirnova, Shishov and Stinebring (1998) 


$$
\tau \propto \theta_{\text {scut }}^{2} \propto\left(\Delta \mathrm{N}_{e}\right)^{\beta},
$$

where

$$
\begin{aligned}
& \beta=2, \mathrm{k} ! \theta_{\text {scat }}<1 \\
& \beta=4 /(\mathrm{n}-2), \mathrm{kl} \theta_{\text {scat }}>1
\end{aligned}
$$

Therefore equation (9) implies a level of turbulence:

$$
\begin{array}{ll}
\delta=\left(\Delta \mathrm{N}_{e} / \mathrm{N}_{e}\right) \propto \mathrm{N}_{e}{ }^{(4-\beta) / \beta}, & \mathrm{N}_{e}<\mathrm{N}_{e, c r i t} \cong 0.7 \mathrm{~cm}^{-3} \\
\delta \propto \mathrm{N}_{e}{ }^{(2-\beta) / \beta}, & \mathrm{N}_{e}>\mathrm{N}_{e, c r i t}, \mathrm{kl} \theta_{\text {scat }}<1 \\
\delta=\mathrm{Const}=\delta_{\text {crit }}, & \mathrm{N}_{e}>\mathrm{N}_{e, c r i t, \mathrm{kl} \theta_{\text {scat }}>1}
\end{array}
$$

This means that turbulence is weak for $\mathrm{N}_{e}<\mathrm{N}_{e, \text { crit }}$. It is possible that turbulence is strong with $\delta \approx 1$ for $\mathrm{N}_{e}>\mathrm{N}_{e, c \text { crit }}$.

\section{Correlation between Distributions in the Galaxy of Pulsars and Plasma Turbulent Clouds}

The results of this section are described in more detail elsewhere (Pynzar' and Shishov, 2001).

Analysis of the distribution of turbulent clouds along the line-of-sight showed that the turbulent plasma is frequently located near pulsars (Smirnova, Shishov and Stinebring, 1998). Therefore one expects an association between pulsars and highdensity turbulent plasma. To check this assumption we investigated the dependence of pulsar number density on the sky, N/S, on emission measure, EM. Here $\mathrm{N}$ is the pulsar number within solid angle $\mathrm{S}$. The statistical data show that the pulsar number density increases with increasing EM up to the value of order of $\mathrm{EM}_{0}=$ $1000 \mathrm{pc} / \mathrm{cm}^{6}$. The characteristic angular scale of an HII region corresponding to $\mathrm{EM}_{0}$ is of order $3^{\circ}$.

To investigate the possible temporal evolution of the pulsar and gas complexes we analyzed the dependence of the emission measure in the direction of the given pulsar, EM, on the pulsar age, T. The distribution of points on the plane $\operatorname{logEM}$ $-\log \mathrm{T}$ shows well defined low boundary $\mathrm{EM}_{\min }$, that decreases with increasing $\mathrm{T}$ for $10^{4}$ years $<\mathrm{T}<10^{6}$ years. This means that pulsars in this boundary region and plasma in the nearby pulsar HII region are burnt at the same time - the time of the supernova burst. Using these values of $\mathrm{EM}_{\min }$ and data for DM one can obtain the dimensions, $\mathrm{R}_{\min }$, of HII regions near pulsars, with ages $\mathrm{T} \sim 10^{4}$ years in the boundary region, and electron densities in these regions

$$
\begin{aligned}
& \mathrm{R}_{\text {min }} \cong 360 \mathrm{pc} \\
& \mathrm{N}_{e, \text { min }} \cong 1.5 \mathrm{~cm}^{-3} .
\end{aligned}
$$

An energy of order $E_{i o n}=10^{52}$ erg is needed for the ionization of such an HII region. The values of emission measure were obtained using the assumption that 
the electron temperature is $\mathrm{T}_{e}=10000^{\circ} \mathrm{K}$. If the temperature is $\mathrm{T}_{e}=10^{6}{ }^{\circ} \mathrm{K}$ one can obtain

$$
\begin{aligned}
& \mathrm{R}_{\text {min }} \cong 70 \mathrm{pc} \\
& \mathrm{N}_{e, \text { min }} \cong 8 \mathrm{~cm}^{-3} .
\end{aligned}
$$

For this case the energy of ionization is of order of $E_{i o n}=5 \cdot 10^{50} \mathrm{erg}$. The problems of the electron temperature in the HII regions and the energy of the ionization should be investigated in more detail.

The data for most pulsars show, on average, decreasing emission measure, EM, with increasing pulsar age, T. The characteristic time of this decrease is of order $\mathrm{T}=10^{6}$ years. It is possible that this time corresponds to the active stage of star formation and supernova events.

We see that a supernova explosion can be an important source of ionization energy for the interstellar plasma. The evolution of an HII region excited by a supernova burst must take into account instability of the cooling plasma. This instability leads to fragmentation of the plasma to dense clouds with the size of order of

$$
\mathrm{L} \approx \mathrm{V}_{s} \cdot \mathrm{T}_{\text {rec }} \approx 0.1-1 \mathrm{pc}
$$

Here $\mathrm{V}_{s}$ is the speed of sound and $\mathrm{T}_{\text {rec }}$ is the recombination time. A series of successive supernova bursts can generate strong plasma turbulence with an outer scale $\mathrm{L}$, because sudden heating leads to expansion of the dense clouds and collisions between them.

\section{Acknowledgements}

This work was supported by Russian Foundation for Basic Research (project num. 00-02-17850 and 00-02-17845), the State Science and Technology Program in Astronomy. The author participation in IAU Colloquium 182 on 'Sources and Scintillations: Refraction and Scattering in Radio Astronomy' was supported by IAU travel grant and by China Local Organizing Committee (Chairperson, Prof. Rendong Nan).

\section{References}

Blanford, M. and Narayan, M.: 1985, Low frequency variability of pulsars, MNRAS 213(3), 591-61 1. Cordes, J.M. and Rickett, B.J.: 1998, Diffractive interstellar scintillation timescales and velocities, Astrophys. J. 502(2), 846-860.

Cordes, J.M., Weisberg, J.M. and Boriakoff, V.: 1985, Small scale electron density turbulence in the interstellar medium, Astrophys. J. 288(10), 221-247.

Martin, J.M. and Flatte, S.M.: 1988, Intensity images and statistics from numerical simulation of wave propagation in 3-D random media, Applied Optics 27(11), 2111-2126. 
Prokhorov, A.M., Bunkin, V.F., Gochelashvily, K.S. and Shishov, V.I.: 1975, Laser irradiance propagation in turbulent media, Proc. IEEE 63(5), 790-811.

Pynzar', A.V. and Shishov, V.I.: 1997, Distribution of turbulent interstellar plasma in the Galaxy, Astron. Rep. 41(5), 663-670.

Pynzar', A.V. and Shishov, V.I.: 1999, Clouds of turbulent interstellar plasma in the spiral arms of the Galaxy, Astron. Rep. 43(7), 504-513.

Pynzar', A.V. and Shishov, V.I.: 2001, Correlation between distributions of pulsars and emission measure in the Galaxy, Astron. Rep. 45(7), 502-503.

Rickett, B.J.: 1969, Frequency structure of pulsar intensity variations, Nature 221(5176), 158-159.

Rickett, B.J., Coles, W.A. and Bourgois, G.: 1984, Slow scintillation in the interstellar medium, $A \& A$ 134(2): 390-395.

Sheuer, P.A.G.: 1968, Amplitude variations in pulsed radio sources, Nature 218(5145): 920-922.

Shishov, V.I.: 1992, Theory of wave scintillations, in: V.I. Tatarski, A. Ishimaru and V.U. Zavorotny (eds.), Waves in Random Media (Scintillation), Invited papers of a conference held 3-7 August 1992, Seattle, Washington, pp. 272-290.

Shishov, V.I.: 1995, Effect of refraction scintillations on the response of an interferometer, WRM 5(4), 497-507.

Sieber, W: 1982, Causal relationship between pulsar long-time intensity variations and the interstellar medium, $A \& A 113(2), 311-313$.

Smirnova, T.V., Shishov, V.I. and Stinebring, D.: 1998, Refractive interstellar scintillations of pulsars, Astron. Rep. 42(6), 766-778.

Stinebring, D.R., Faison, M.D. and McKinnon, M.M.: 1996, Refractive and diffractive scintillation of the pulsar PSR B0329+54, Astrophys. J. 460(1), 460-469. 